\title{
LA-UR-15-23358
}

Approved for public release; distribution is unlimited.

Title: $\quad$ Calculations in Support of JAEA ZEUS Experiments

Author(s): James, Michael R.

Intended for: Report

Issued: 
Disclaimer:

Los Alamos National Laboratory, an affirmative action/equal opportunity employer,is operated by the Los Alamos National Security, LLC for the National NuclearSecurity Administration of the U.S. Department of Energy under contract DE-AC52-06NA25396. By approving this article, the publisher recognizes that the U.S. Government retains nonexclusive, royalty-free license to publish or reproduce the published form of this contribution, or to allow others to do so, for U.S. Government purposes. Los Alamos National Laboratory requests that the publisher identify this article as work performed under the auspices of the U.S. Departmentof Energy. Los Alamos National Laboratory strongly supports academic freedom and a researcher's right to publish; as an institution, however, the Laboratory does not endorse the viewpoint of a publication or guarantee its technical correctness. 
- Los Alamos

NATIONAL LABORATORY

EST. 1943

\section{Calculations in Support of JAEA ZEUS Experiments}

\section{Michael James, Los Alamos National Laboratory}

April 23, 2015 
This is to report recent calculations in support of the experiments on ZEUS to measure $\mathrm{Pb}$ void reactivity.

In this package are LANL simulations which are compared to results reported in "Analysis of Comet HEU/Lead experiment" from the JAEA

\section{Analysis of Comet HEU/Lead experiments}

$$
\text { Japan Atomic Energy Agency (JAEA) }
$$

Research Group for Nuclear Transmutation System 


\section{LANL model}

This configuration is the most recent representation for the expected ZEUS HEU critical assembly.

The $\mathrm{Pb}$ plates are $1.2 \mathrm{~cm}$ thick. But each of these are composed of two $0.594 \mathrm{~cm}$ thick $\mathrm{Pb}$ plates. Of this, $0.442 \mathrm{~cm}$ is $\mathrm{Pb}$ with $0.07874 \mathrm{~cm}$ Al clad on top and bottom.

Therefore, the $\mathrm{Pb} / \mathrm{Al}$ is about $73.7 \%$ $\mathrm{Pb}$ and $26.3 \% \mathrm{Al}$ fraction by volume (92.2 at.\% Pb, 7.8 at.\% Al).

The homogenized density is $9.016 \mathrm{~g} / \mathrm{cm}^{3}$.

This configuration was run with

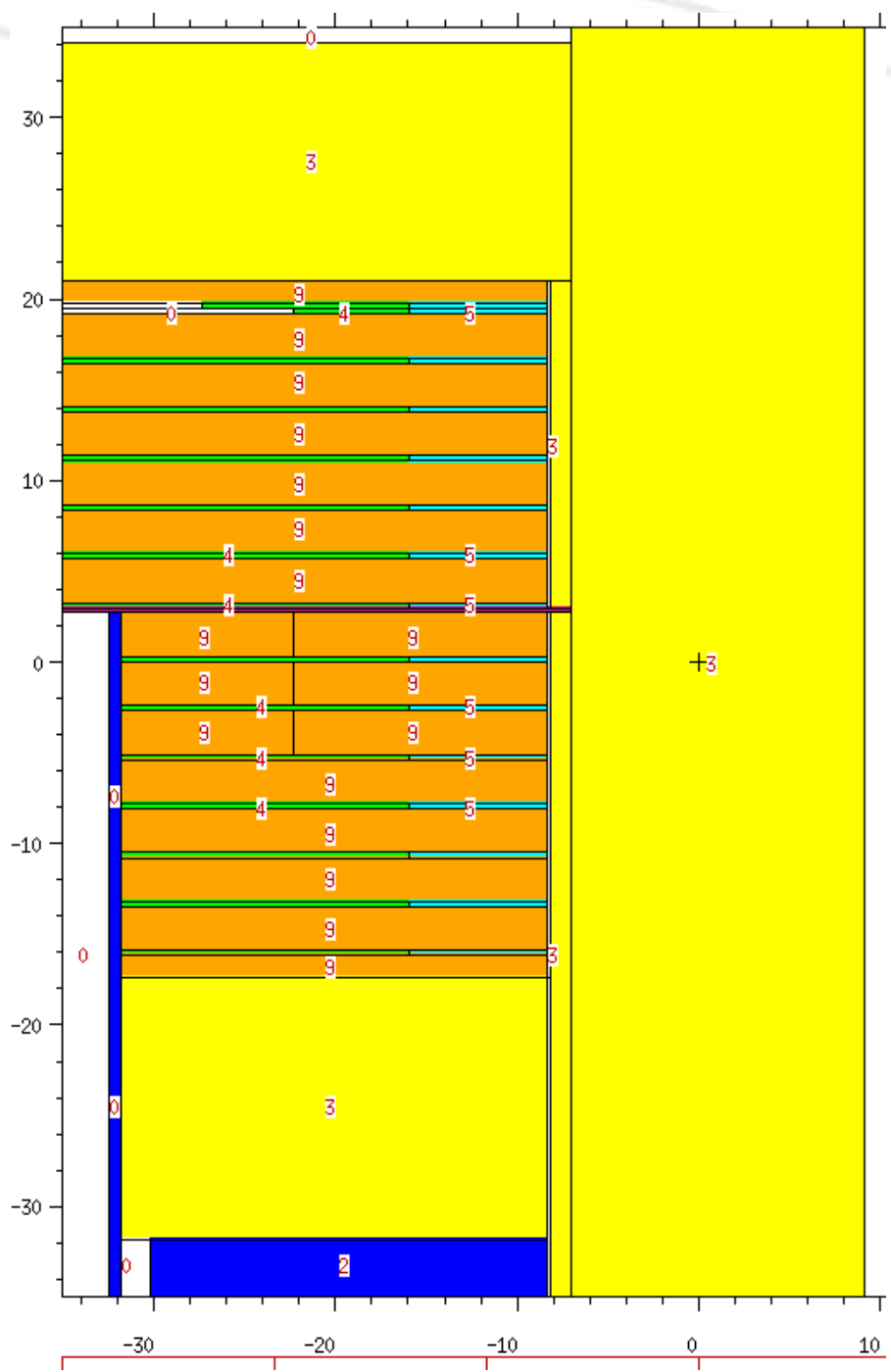
ENDF 7.1 and JENDL 4.0 libraries. 


\section{JAEA vs LANL model}
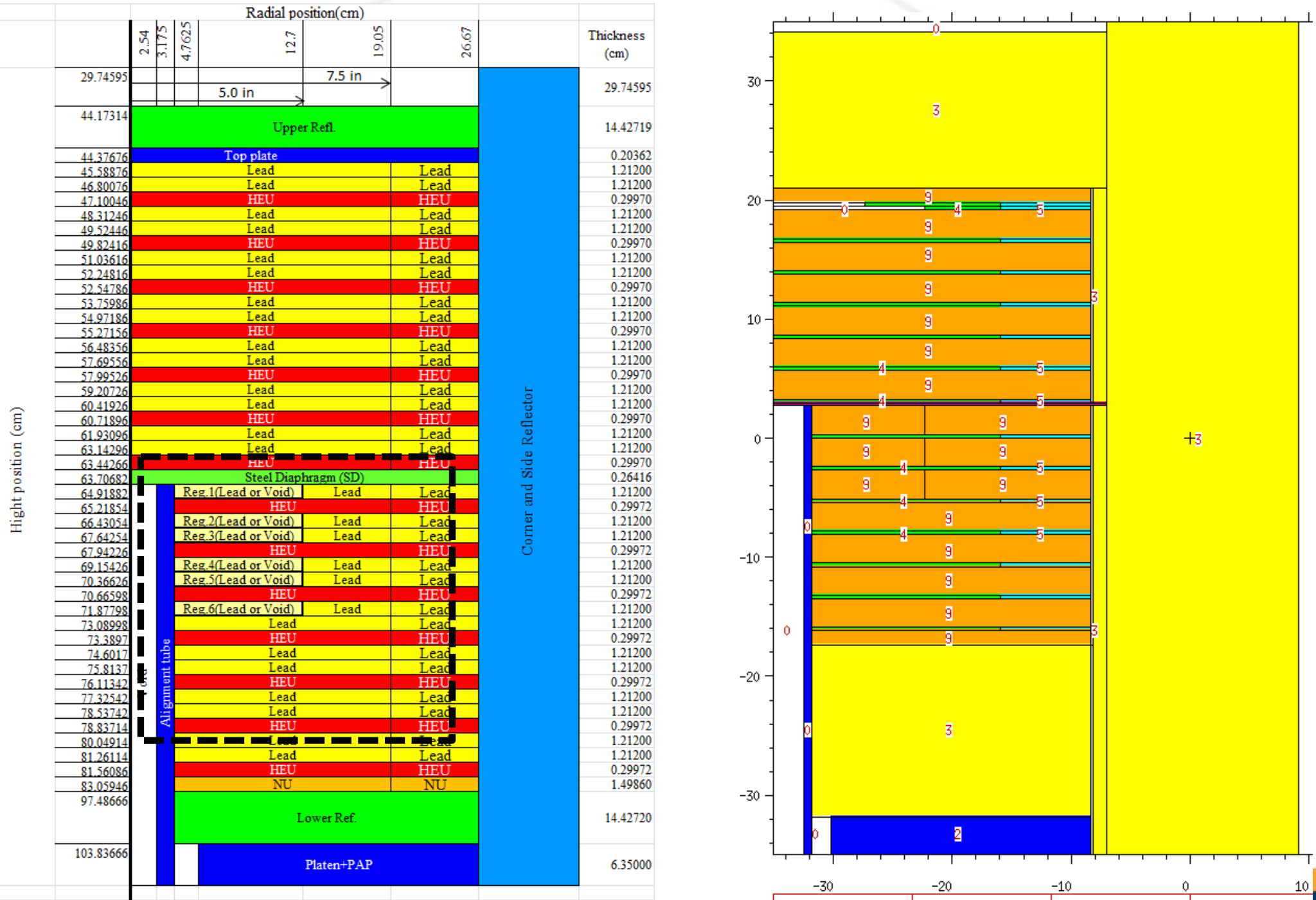

UNCLASSIFIED 


\section{JAEA vs LANL model}

Comparison:

- Stacking of HEU/Pb slightly off based on different "unit stack" composition.

- LANL model has no "top plate"

- LANL model does not have lower "NU" plate

- Small difference in Pb/Al plates: 1.212 (JAEA) cm vs 1.2cm (LANL).

- Material uncertainties

- Composition of $\mathrm{Pb}$ is the largest uncertainty. Other issues could be in composition of upper reflector, lower reflector and corner and side reflectors. 


\section{$\mathrm{K}_{\text {eff }}$ Comparison: JAEA Results vs. LANL Model Runs}

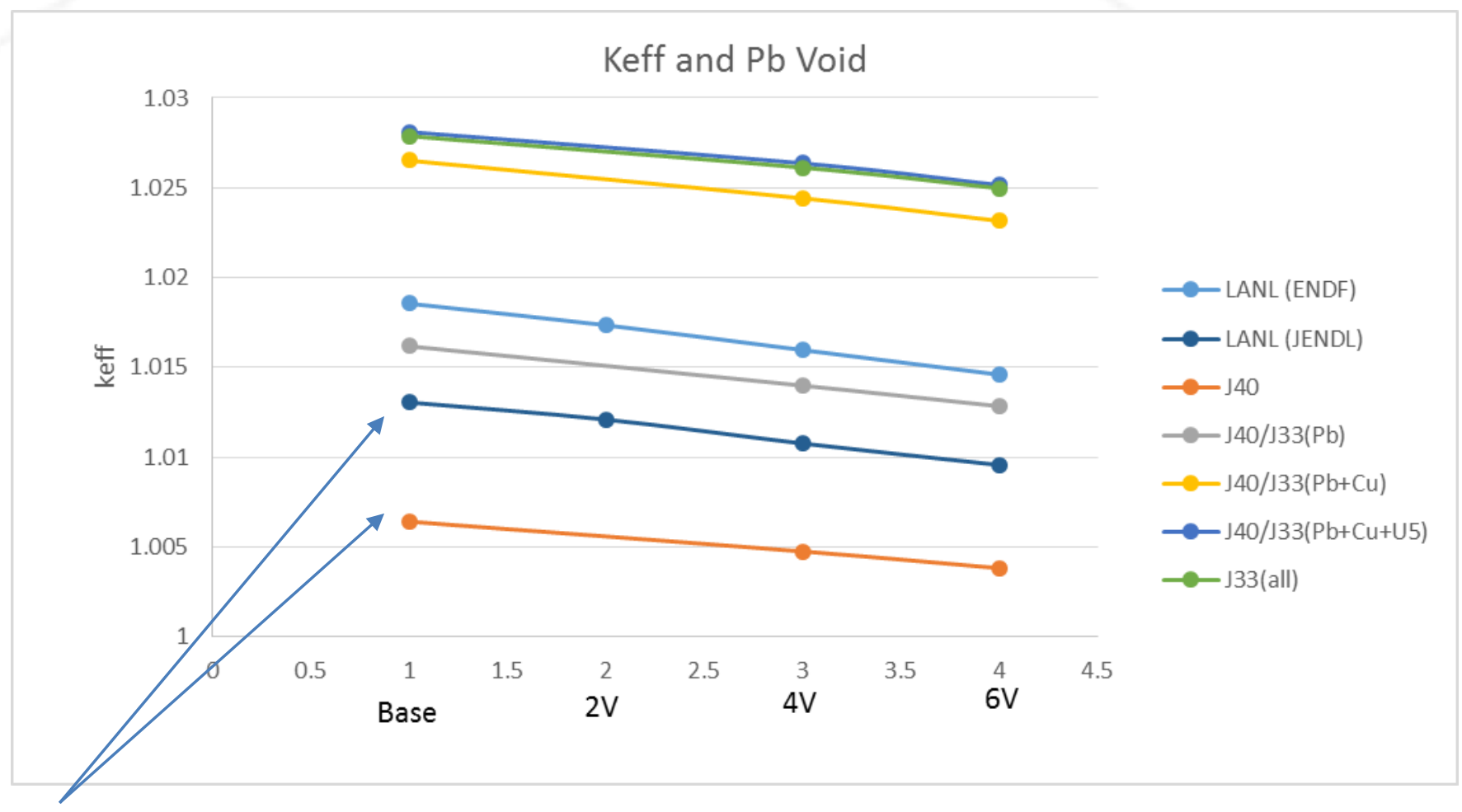

Same nuclear data, difference due to geometry? 


\section{Pb Void Reactivity Change}

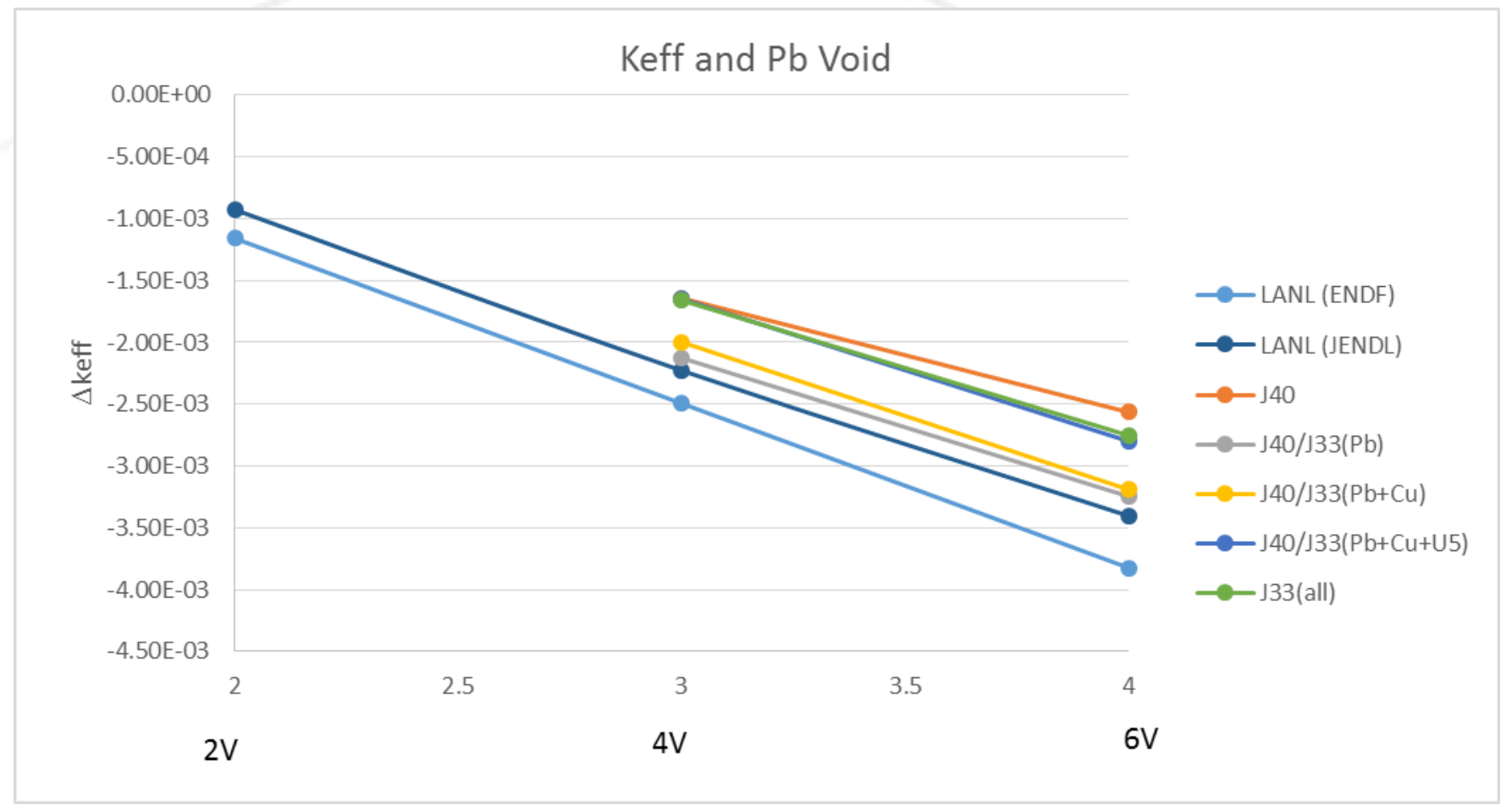

Overall, Japanese expectation of $\mathrm{Pb}$ void reactivity are smaller in magnitude than LANL simulations.

JENDL40 has smaller Pb void worth than ENDF or JENDL33. Note: $\Delta \mathrm{k}_{\text {eff }}=\frac{k_{2}-k_{1}}{k_{2} * k_{1}}$ is used to match results reported in JAEA slides.

UNCLASSIFIED 


\section{KSEN Coefficients for Pb, JENDL vs. ENDF}

\begin{tabular}{|c|c|c|c|c|c|c|c|}
\hline JENDL4 & & & & ENDF7.1 & & & \\
\hline isotope & reaction & sensitivity & rel unc & isotope & reaction & sensitivity & rel unc \\
\hline $82204.40 c$ & total + sab & 1.62E-04 & 1.7711 & $82204.80 c$ & total + sab & 9.46E-04 & 0.3282 \\
\hline $82204.40 c$ & capture & $-8.66 \mathrm{E}-04$ & 0.0019 & $82204.80 c$ & capture & $-4.72 \mathrm{E}-04$ & 0.0024 \\
\hline $82204.40 c$ & elastic & 5.99E-04 & 0.471 & $82204.80 c$ & elastic & 9.85E-04 & 0.3101 \\
\hline $82204.40 c$ & inelastic & 4.26E-04 & 0.1417 & $82204.80 c$ & inelastic & 4.27E-04 & 0.138 \\
\hline $82206.40 c$ & total + sab & 2.18E-02 & 0.0522 & $82206.80 c$ & total + sab & 2.01E-02 & 0.0569 \\
\hline $82206.40 c$ & capture & $-2.00 \mathrm{E}-03$ & 0.0033 & $82206.80 c$ & capture & $-1.97 \mathrm{E}-03$ & 0.0033 \\
\hline $82206.40 c$ & elastic & 1.75E-02 & 0.0632 & $82206.80 c$ & elastic & 1.54E-02 & 0.0725 \\
\hline $82206.40 c$ & inelastic & $6.18 \mathrm{E}-03$ & 0.04 & $82206.80 c$ & inelastic & $6.53 E-03$ & 0.0377 \\
\hline $82207.40 c$ & total + sab & $2.05 E-02$ & 0.0565 & $82207.80 c$ & total + sab & 2.17E-02 & 0.0545 \\
\hline $82207.40 c$ & capture & -1.07E-03 & 0.0033 & $82207.80 c$ & capture & $-1.08 E-03$ & 0.0032 \\
\hline $82207.40 c$ & elastic & 1.60E-02 & 0.0713 & $82207.80 c$ & elastic & 1.66E-02 & 0.0697 \\
\hline $82207.40 c$ & inelastic & 5.33E-03 & 0.0454 & $82207.80 c$ & inelastic & 5.93E-03 & 0.0414 \\
\hline $82208.40 c$ & total + sab & 4.26E-02 & 0.0416 & $82208.80 c$ & total + sab & 4.31E-02 & 0.0409 \\
\hline $82208.40 c$ & capture & $-3.52 E-04$ & 0.0018 & $82208.80 c$ & capture & $-4.97 \mathrm{E}-04$ & 0.0039 \\
\hline $82208.40 c$ & elastic & 4.02E-02 & 0.0439 & $82208.80 c$ & elastic & 4.07E-02 & 0.0432 \\
\hline $82208.40 c$ & inelastic & 2.35E-03 & 0.0663 & $82208.80 c$ & inelastic & $2.45 \mathrm{E}-03$ & 0.0647 \\
\hline
\end{tabular}


The HEU-MET-FAST-072-001 benchmark was used to check that the LANL MCNP6/JENDL4 libraries give consistent results to JAEA.

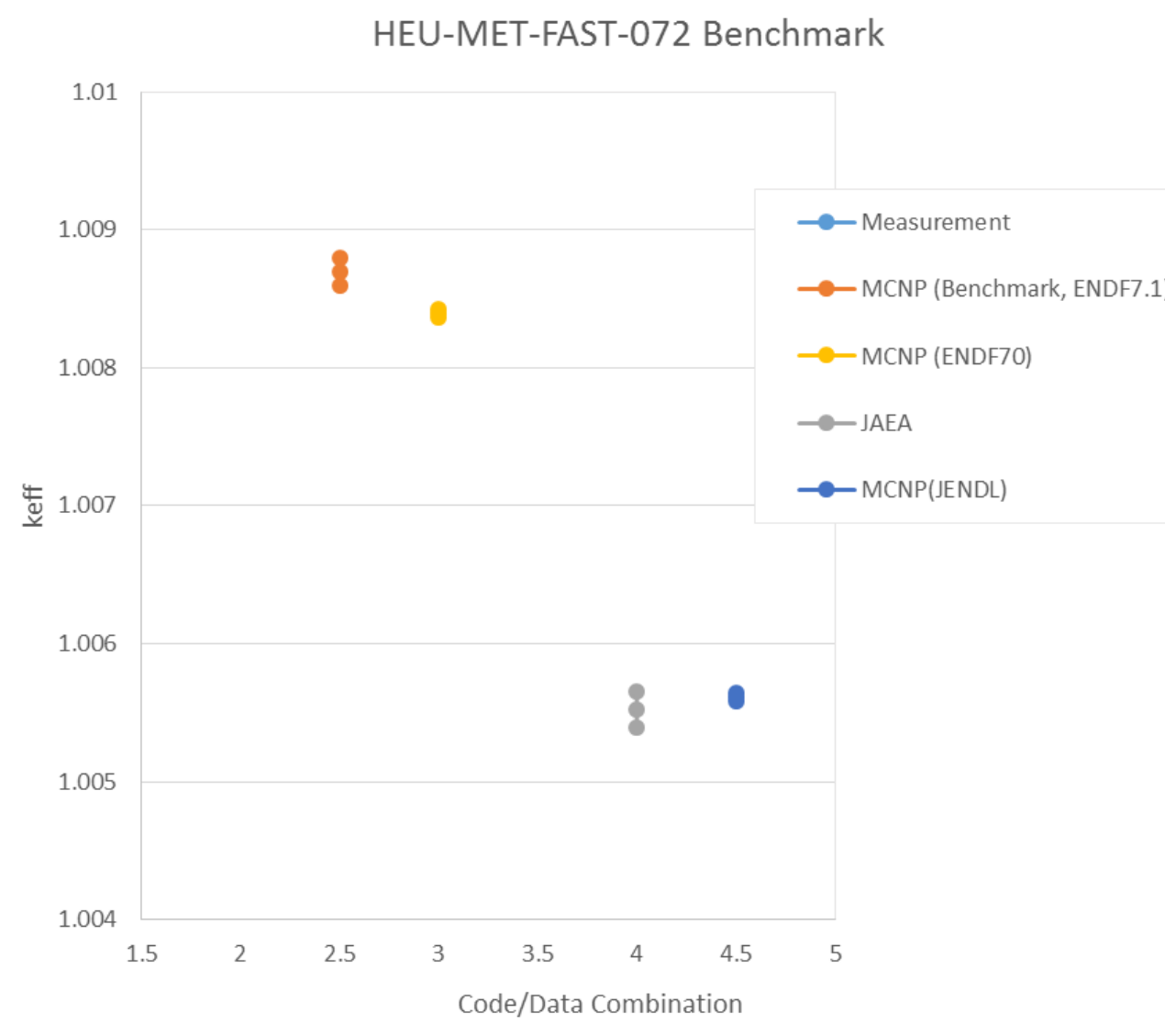

\begin{tabular}{|l|r|r|}
\hline Case & \multicolumn{1}{|c}{ keff } & 1-sigma \\
\hline Measurement & 0.9991 & 0.0024 \\
\hline MCNP (Benchmark, ENDF7.1) & 1.0087 & 0.0001 \\
\hline JAEA & 1.00552 & 0.00013 \\
\hline MCNP (ENDF70) & 1.0084 & 0.00003 \\
\hline MCNP(JENDL) & 1.00561 & 0.00003 \\
\hline
\end{tabular}

Results: LANL results match JAEA results with our versions of MCNP and JENDL-4.0 nuclear data. Differences in ZEUS runs must be in geometry/materials 


\section{JAEA Comparison}

Reconfigured LANL ZEUS model to look like JAEA-reported configuration
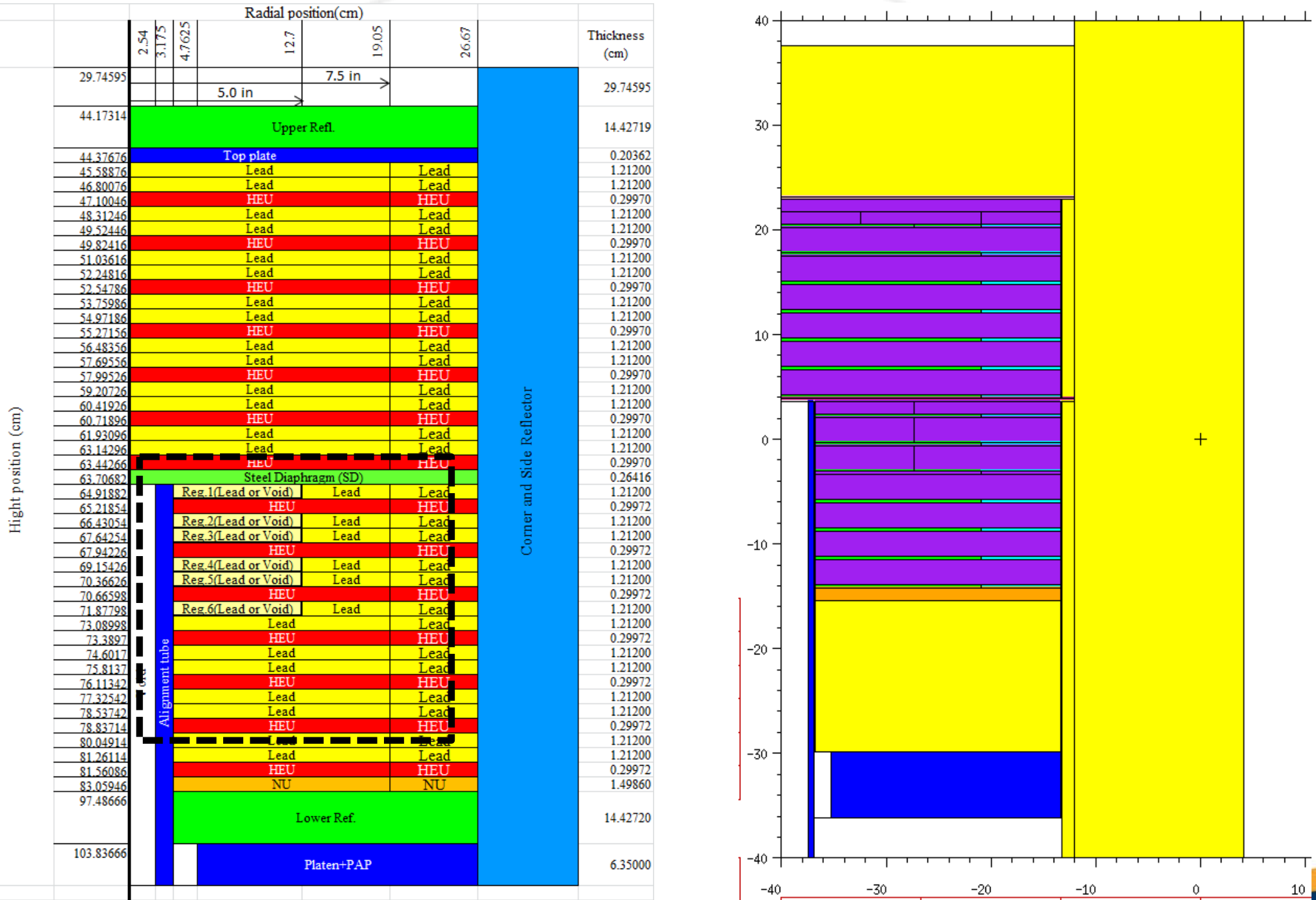

UNCLASSIFIED 
Duplicated the stacking, thicknesses of $\mathrm{Pb}$ plates, presence of $\mathrm{NU}$ and other plates from the JAEA representation.

Used JENDL4.0 cross sections for calculations.

Composition of $\mathrm{Pb}$ is unknown. Three runs were made: one with normally

diluted ( $\mathrm{Pb}-7.8$ at\% Al mixture), one with $\mathrm{Pb}-0.2$ at\% $\mathrm{Al}$ (which represents $0.012 \mathrm{~cm} \mathrm{Al}$ on $1.2 \mathrm{~cm}$ of $\mathrm{Pb}$ ) the other $100 \% \mathrm{~Pb}$.

\section{Results:}

$\mathrm{Pb}: 7.8 \mathrm{Al}$

1.01507

$\mathrm{Pb}: 0.2 \mathrm{Al}$

1.00855

$\mathrm{Pb}$

1.00894

JAEA

1.00643

Conclusion: JAEA results are outside of bounds these calculations.

Are there other variations in material composition or density $(\mathrm{Pb}, \mathrm{Cu})$ ? 
Further calculations were performed with lower values for density of Pb/Al.

Rho $=8.4 \mathrm{~g} / \mathrm{cm}^{3}$ is a good fit for the base case (actual den $=9.016$ ).

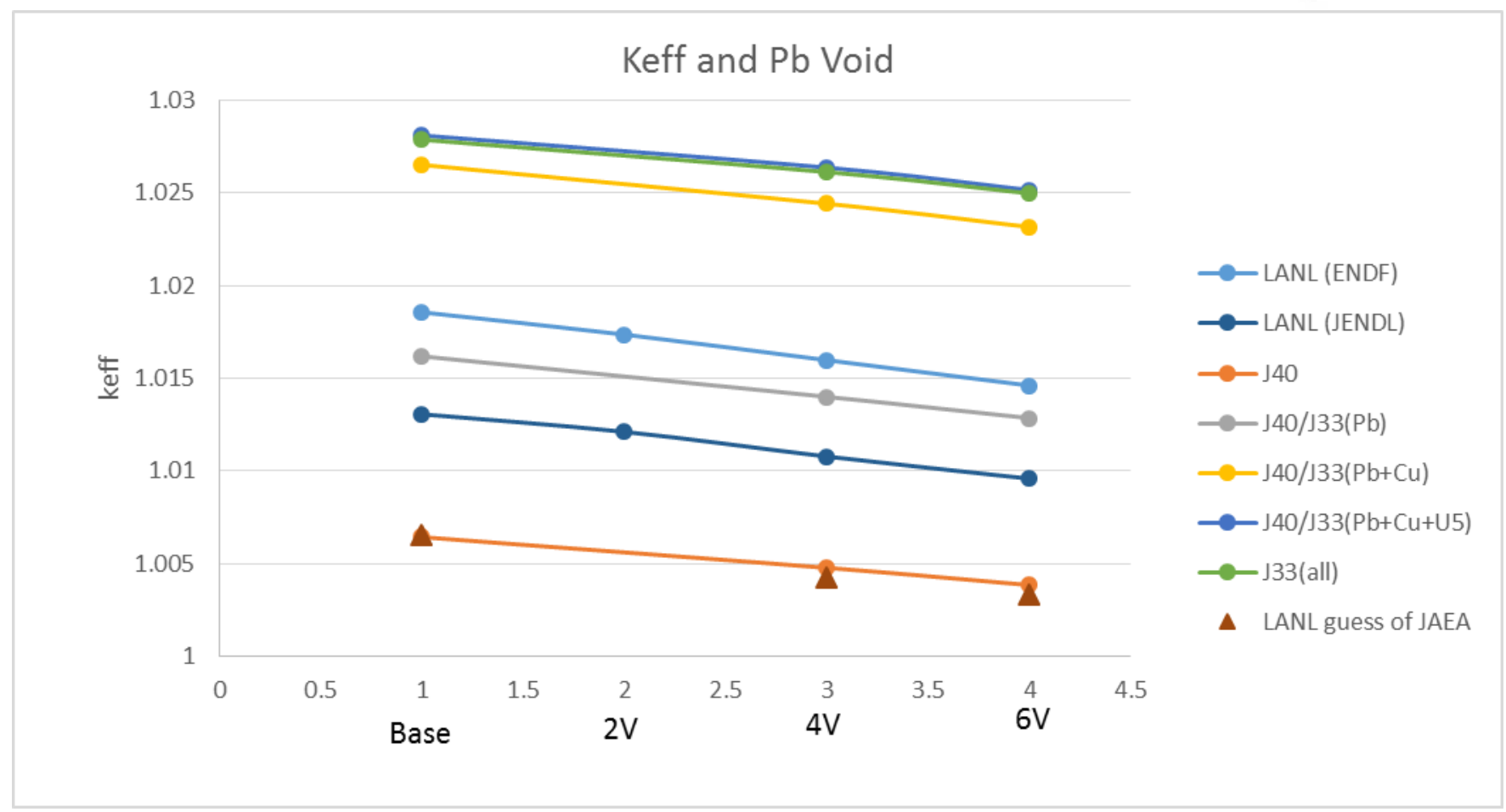

UNCLASSIFIED 


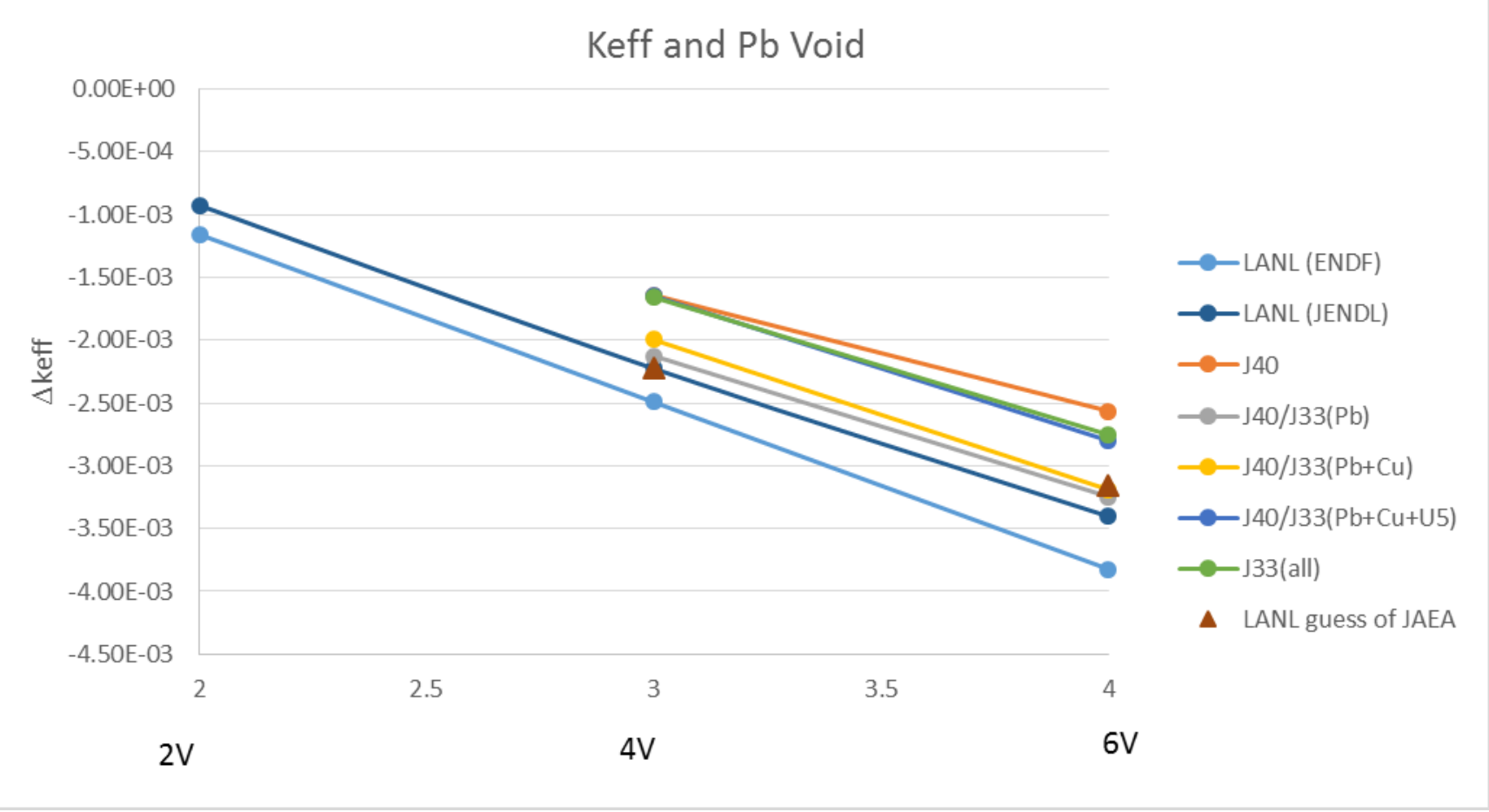

But reactivity change from voided $\mathrm{Pb}$ regions still does not match JAEA results? 
Conclusions:

LANL calculations tend to support Pb void worth of $-3.40 \mathrm{E}-03$ to $-3.80 \mathrm{E}-03$ when all $\mathrm{Pb}$ plates are removed (6V).

JAEA calculations reported show void worth of $-2.56 \mathrm{E}-03$ to $-3.25 \mathrm{E}-03$ depending on choice of libraries.

LANL simulations expect base keff $=1.0131(1)$ to $1.0186(1)$ while JAEA results are between 1.00643(5) to 1.02811(5).

It is still not clear why such large discrepancies still exists although it seems likely the models are different in a fundamental way. 


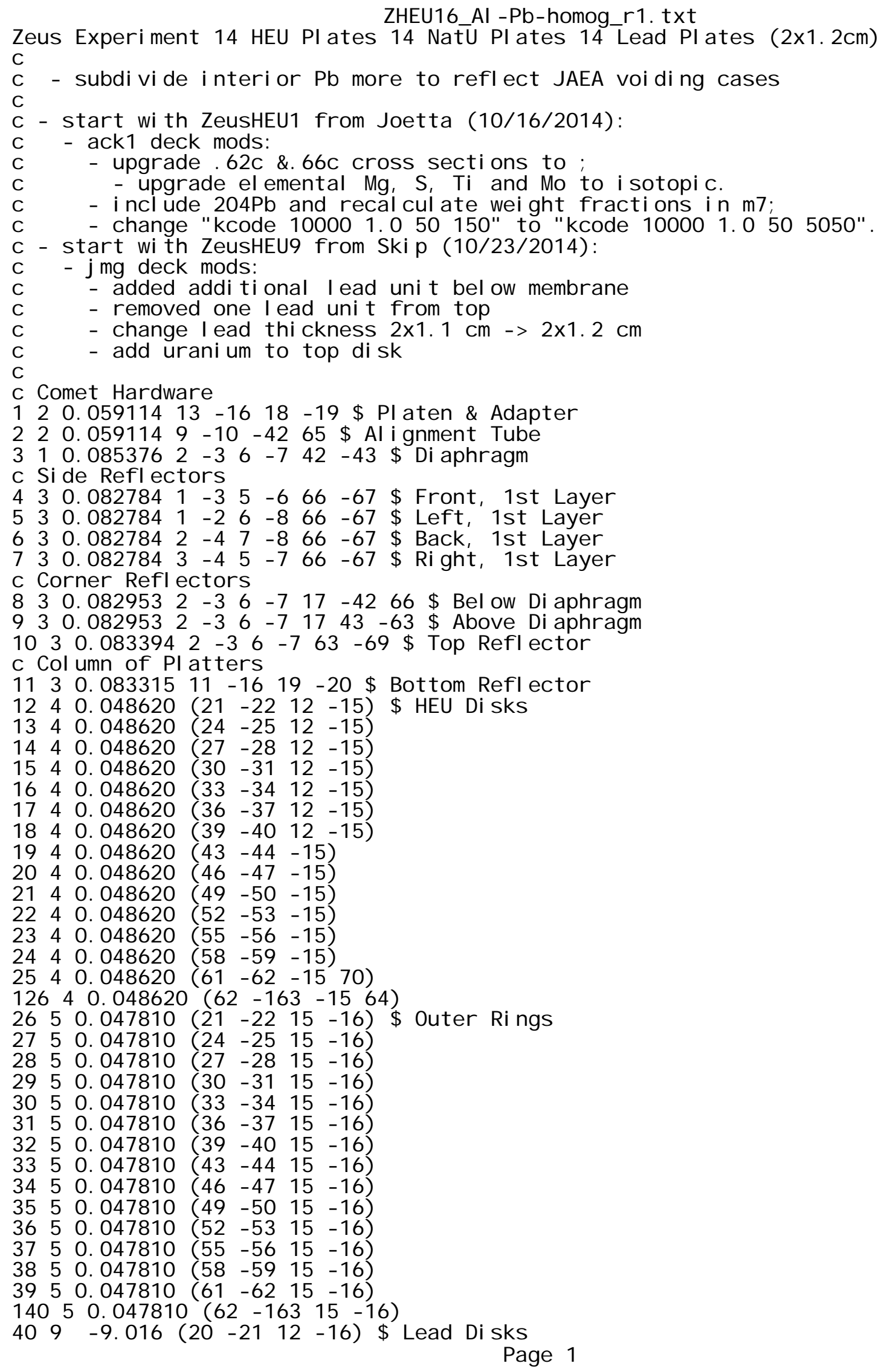




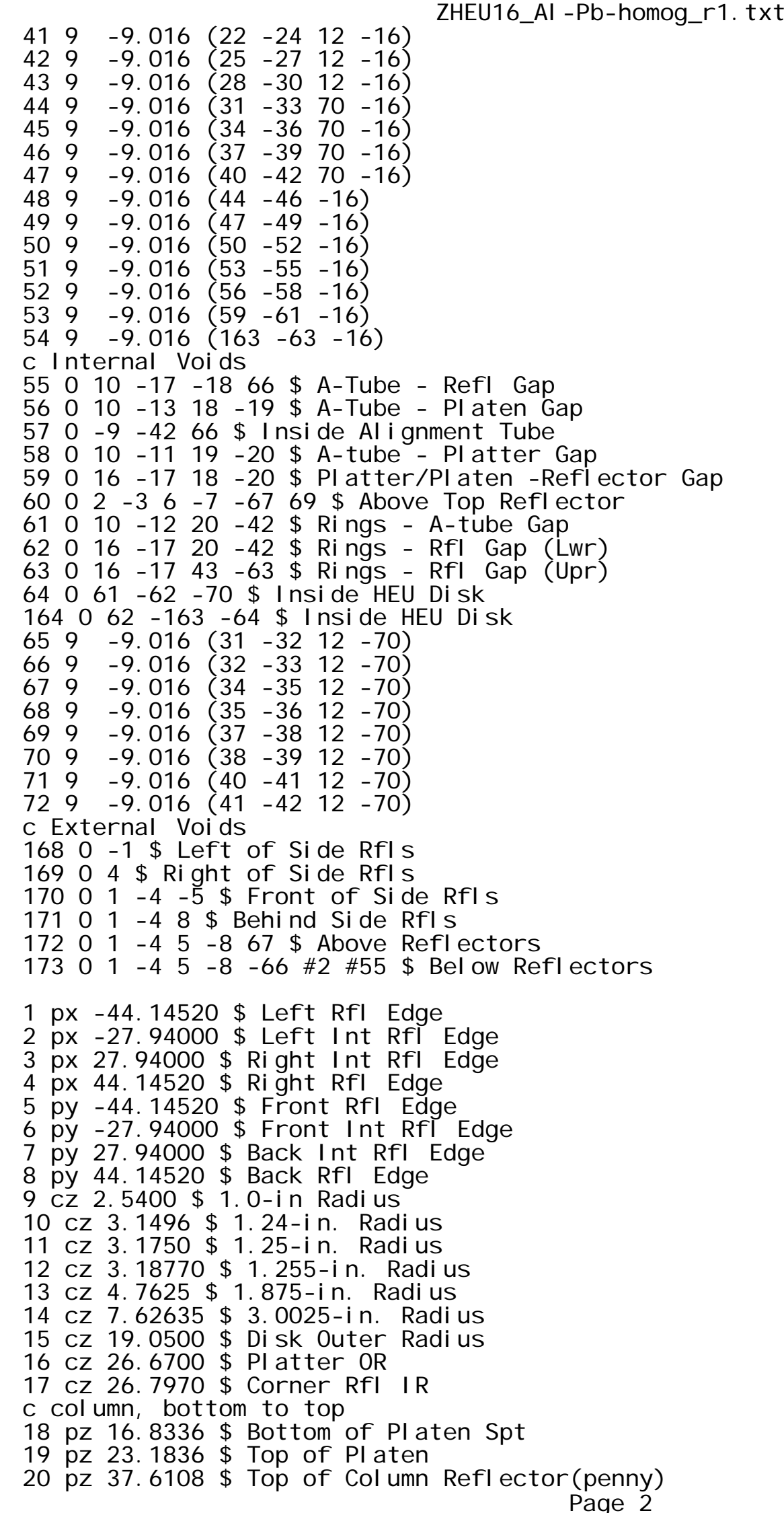


ZHEU16 Al-Pb-homog_r 1.t xt

$21 \mathrm{pz} 38.8108$ \$NatU Disk 1 with Wi de Hole

$22 \mathrm{pz} 39.1105$ \$ HEU Disk and Ring 1

$23 \mathrm{pz} 40.3105$ \$ Lead Disk 1

$24 \mathrm{pz} 41.5105$ \$ Lead Disk 1

$25 \mathrm{pz} 41.8102$ \$ HEU Disk and Ring 2

$26 \mathrm{pz} 43.0102$ \$ Lead Disk 2

$27 \mathrm{pz} 44.2102$ \$ Lead Disk 2

$28 \mathrm{pz} 44.5099$ \$ HEU Disk and Ring 3

$29 \mathrm{pz} 45.7099$ \$ Lead Disk 3

$30 \mathrm{pz} 46.9099$ \$ Lead Disk 3

$31 \mathrm{pz} 47.2096$ \$ HEU Disk and Ring 4

$32 \mathrm{pz} 48.4096$ \$ Lead Disk 4

$33 \mathrm{pz} 49.6096$ \$ Lead Disk 4

$34 \mathrm{pz} 49.9094$ \$ HEU Disk and Ring 5

$35 \mathrm{pz} 51.1094$ \$ Lead Disk 5

$36 \mathrm{pz} 52.3094$ \$ Lead Disk 5

$37 \mathrm{pz} 52.6091$ \$ HEU Disk and Ring 6

$38 \mathrm{pz} 53.8091$ \$ Lead Disk 6

$39 \mathrm{pz} 55.0091$ \$ Lead Disk 6

$40 \mathrm{pz}$ 55.3088 \$ HEU Disk and Ring 7

$41 \mathrm{pz} 56.5088$ \$ Lead Disk 7

$42 \mathrm{pz} 57.7088$ \$ Lead Disk 7

43 pz 57.97296\$Di aphragm

$44 \mathrm{pz} 58.2727$ \$ HEU Disk and Ring 1

$45 \mathrm{pz} 59.4727$ \$ Lead Disk 1

$46 \mathrm{pz} 60.6727$ \$ Lead Disk 1

$47 \mathrm{pz} 60.9724$ \$ HEU Disk and Ring 2

$48 \mathrm{pz} 62.1724$ \$ Lead Disk 2

$49 \mathrm{pz} 63.3724$ \$ Lead Disk 2

$50 \mathrm{pz}$ 63.67212 \$ HEU Disk and Ring 3

$51 \mathrm{pz} 64.87212$ \$ Lead Disk 3

$52 \mathrm{pz} 66.07212$ \$Lead Disk 3

$53 \mathrm{pz}$ 66.37184 \$HEU Disk and Ring 4

54 pz 67.57184 \$ Lead Disk 4

$55 \mathrm{pz} 68.77184$ \$Lead Disk 4

$56 \mathrm{pz} 69.07156$ \$ HEU Disk and Ring 5

$57 \mathrm{pz} 70.27156$ \$ Lead Disk 5

$58 \mathrm{pz} 71.47156$ \$Lead Disk 5

$59 \mathrm{pz} 71.77128$ \$ HEU Disk and Ring 6

$60 \mathrm{pz} 72.97128$ \$ Lead Disk 6

$61 \mathrm{pz} 74.17128$ \$ Lead Disk 6

$62 \mathrm{pz} 74.471$ \$ HEU Ring 7

$163 \mathrm{pz} 74.7707$ \$ HEU Ring 8

$63 \mathrm{pz} 75.971$ \$ Lead Disk 7

64 Cz 7.62 \$6.in Radius

c support structure

$65 \mathrm{pz}-5.79120$ \$Bottom of Alignment Tube

$66 \mathrm{pz} 0.0$ \$ Bottom of Reflectors

c side reflectors

$67 \mathrm{pz} 103.25100$ \$ Top of Side Reflectors

c corner and top reflectors

$68 \mathrm{pz} 75.471$ \$ Top of Corner Reflectors and Lead Disk 7

$69 \mathrm{pz} 89.0979$ \$ Top Reflector

$70 \mathrm{cz} 12.7$ \$ Lead/Void cylinder surface (5-in Radius)

mode $n$

kcode 50001.010010100

rand hist $=14285714$

i mp:n 1.0 $74 \mathrm{r} 0.05 \mathrm{r}$

ksrc $0.0-15.041 .460620 .0 \quad 15.041 .46062$

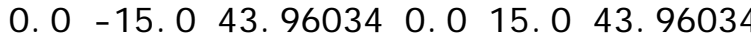

$0.0-15.0 \quad 46.46006 \quad 0.0 \quad 15.046 .46006$

$0.0-15.0 \quad 48.95978 \quad 0.0 \quad 15.0 \quad 48.95978$

Page 3 


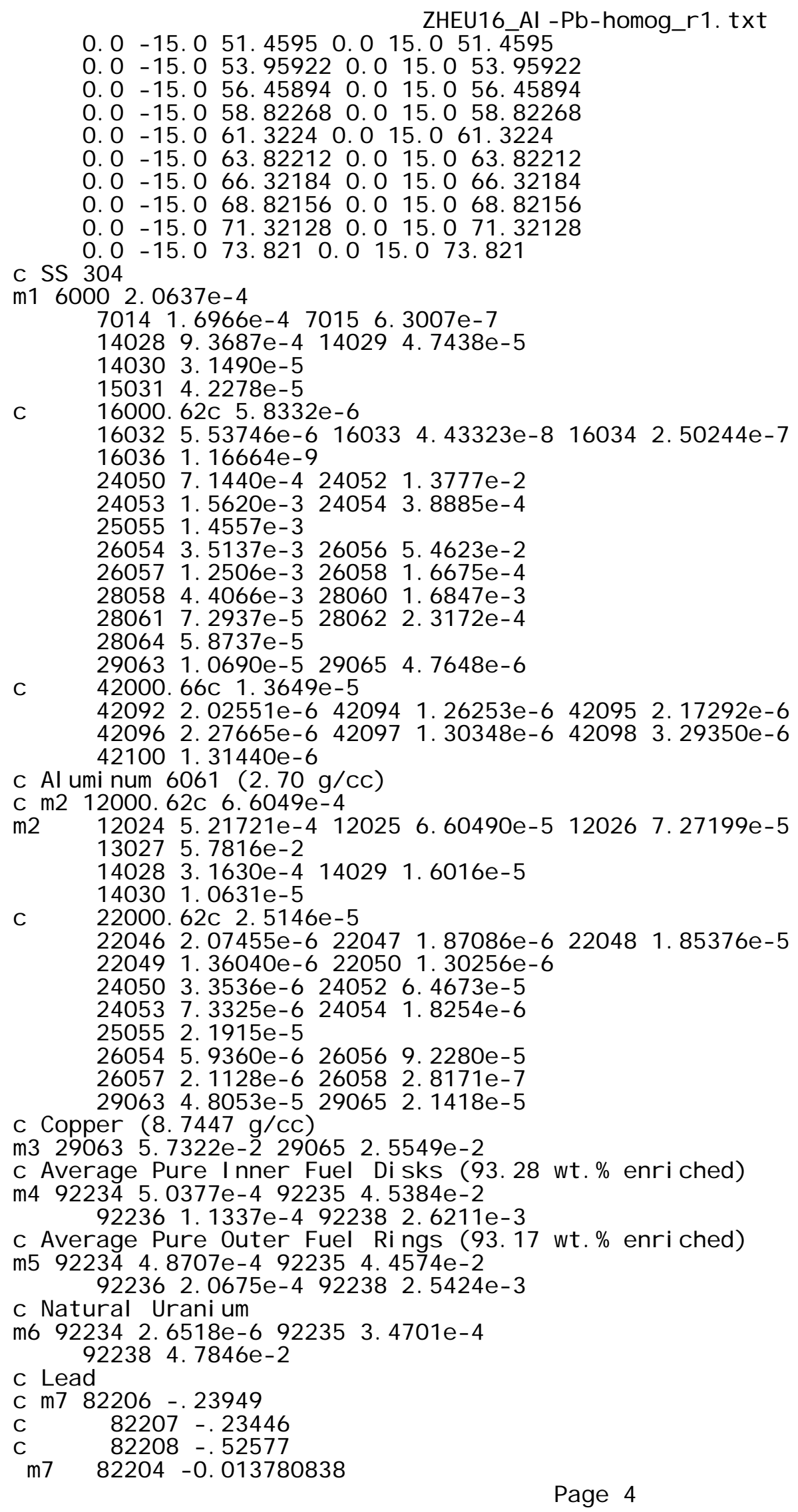




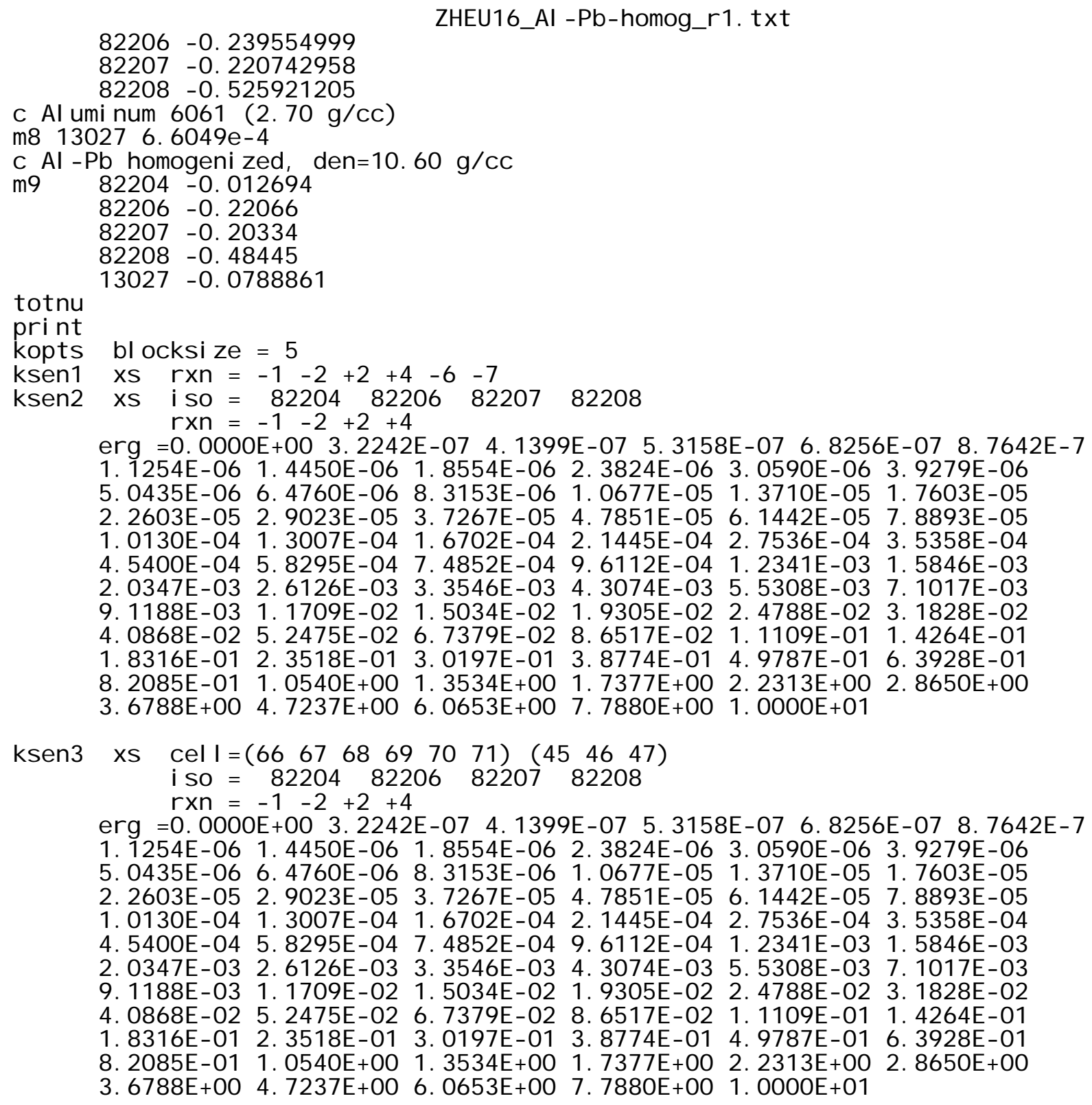

